\title{
Entwicklung eines Metamodells zur Modellierung der fachlichen Fähigkeiten der Softwarearchitektur in Krankenhäusern auf Grundlage von Stakeholder Concerns
}

\author{
René Fitterer, Peter Rohner \\ Institut für Wirtschaftsinformatik, \\ Universität St. Gallen
}

\section{Einleitung}

Das Management von Unternehmensarchitekturen (EAM) als ganzheitlicher Ansatz zur Analyse und Gestaltung von Organisationen wird in zunehmendem Maße in Unternehmen eingesetzt. Die Unternehmensarchitektur (EA) ist definiert als die fundamentale Strukturierung einer Organisation (Unternehmen, Behörde, etc.) durch Abbildung der wichtigsten Artefakte (Gestaltungsgegenstände, Abhängigkeiten, etc.) (Aier et al. 2008b, S. 292). Eine Abbildung dieser Artefakte in Form von Modellen verfolgt das Ziel der Explikation der fundamentalen Strukturierung der Organisation, welche zu Analysezwecken und als Entscheidungsgrundlage für die Gestaltung der EA dient. Zur Darstellung und Modellierung der EA werden in verschiedenen Beiträgen von Wissenschaftlern und Praktikern zahlreiche Frameworks definiert (z. B. CIO-Council 1999; Goethals et al. 2006; Ifip-Ifac 1998; Zachman 1987). Im Sinne eines ganzheitlichen und systematischen Vorgehens integriert die EA Modelle der Ebenen Geschäftsarchitektur (Strategie, Ziele, etc.), Prozessarchitektur (Ablauf- und Aufbauorganisation, etc.), Integrationsarchitektur (sozio-technische Informationssysteme - IS), Softwarearchitektur (Softwarekomponenten, Datenstrukturen, etc.) und Technologiearchitektur (Hardware, Netzwerk, etc.) (The Open Group 2009; Winter und Fischer 2007), um eine konsistente und zielgerichtete Veränderung der Organisation zu unterstützen.

Eine empirische Untersuchung zur Bedeutung und Umsetzung der Gestaltungsobjekte auf den genannten Ebenen des EAM zeigt jedoch, dass einerseits strategiebezogene Gestaltungsobjekte oftmals kein expliziter Bestandteil bzw. darin nur sehr schwach ausgeprägt sind (Aier et al. 2008b, S. 294) und andererseits die methodische Unterstützung von Analysen und Einsatzszenarien der EA vielfach vernachlässigt wird (Aier et al. 2008b, S. 300). EAM bleibt in vielen Unternehmen somit auf die ,strukturierte und aufeinander abgestimmte Sammlung von Plänen 
für die Gestaltung der IT-Landschaft eines Unternehmens, die in verschiedenen Detaillierungen und Sichten, ausgerichtet auf spezielle Interessengruppen, unterschiedliche Aspekte von IT-Systemen und deren Einbettung in das Geschäft [..] darstellen“, beschränkt (Niemann 2005, S. 21). Insbesondere für Organisationen, die eine geringe Reife bezüglich des EAM aufweisen, besteht auf Grund der Komplexität der Artefakte des EAM die Herausforderung, den Betrachtungshorizont (Breite und Tiefe) auf die relevanten Aspekte (im Folgenden auf Basis des originalen englischen Begriffs: Concerns) der EA-Stakeholder zu reduzieren (Kurpjuweit und Winter 2007; Sutton und Rouvellou 2001). Dieser Artikel beschreibt am Beispiel von Krankenhäusern, die auf Grund der Integration diverser Berufsgruppen und der fragmentierten, spezialisierten Leistungserstellung als komplexe Organisationen (ähnlich einem großen Unternehmen in anderen Branchen) erachtet werden können, die Entwicklung eines Metamodells zur Modellierung der fachlichen Fähigkeiten (engl. Capabilities) der Softwarearchitektur auf Grundlage von Stakeholder Concerns. Der folgende Abschnitt gibt einen kurzen Überblick der bestehenden Arbeiten zum EAM und deren Anwendbarkeit auf die adressierte Problemstellung, sowie eine kurze Beschreibung der Herausforderungen des EAM in Krankenhäusern. Anschließend präsentiert der Artikel die Entwicklung eines Metamodells zur Modellierung der relevanten Concerns in Krankenhäusern und beschreibt kurz dessen Evaluierung im Rahmen einer Studie zum Benchmarking der Softwarearchitekturen von 13 Schweizer Krankenhäusern. Abschließend werden die beschrieben Ergebnisse kritisch diskutiert und ein Ausblick auf weitergehende Forschungsarbeiten gegeben.

\section{Grundlagen}

\subsection{EAM Artefakte}

Als Design Research Artefakte verfolgen EAM-Methoden und -Modelle das Ziel der Lösung relevanter Probleme der Praxis (Frank 2000; Hevner et al. 2004; March und Smith 1995). Die bestehenden Ansätze adressieren unterschiedliche Gestaltungsziele (Aier und Gleichauf 2009; Aier et al. 2008a; Buchanan und Soley 2002; Veasey 2001; Winter und Fischer 2007):

- strategische und organisatorische Ziele sowie deren Veränderungen adäquat in IS-Architektur und IT-Infrastruktur (IT follows business) abzubilden,

- organisatorische Veränderungen auf Grund von technischen Innovationen konsistent umzusetzen (IT als Enabler) und

- fachliche Anforderungen und technische Möglichkeiten der IS (IT/Business Alignment) abzugleichen.

Im Sinne des IT follows business (i) ermöglicht ein an der Geschäftsarchitektur ausgerichtetes EAM eine Einschränkung des Betrachtungsraums von EA- 
Modellen auf relevante fachliche Gestaltungsobjekte (Strategie, Stakeholder, Prozesse, Aufbauorganisation). Insbesondere im Rahmen eines strategiebezogenen EAM bedarf es hierbei nur bedingt einer umfänglichen Analyse der Software- und Technologiearchitektur (Systemschnittstellen, Workflows, etc.), sondern vielmehr eines Fokus auf die fachlichen Ebenen der EA und der entsprechenden Verknüpfung mit den IT-Komponenten im Rahmen der Integrationsarchitektur. Einige Frameworks wie beispielsweise TOGAF unterstützen den Top Down-Ansatz des IT follows Business-Designparadigmas. Andere Ansätze liefern eine Reihe von Analysen, die für die Einschätzung des Beitrags der Softwarearchitektur zur Unterstützung oder Verbesserung der Geschäftsarchitektur verwendet werden können (Lankhorst 2005). Die beschriebenen Frameworks definieren jedoch in der Regel lediglich generische Modellierungsansätze, beispielsweise zur Abdeckungsanalyse (Aier et al. 2008b).

Entsprechend besteht für EA, als Entscheidungsunterstützungssystem des Chief Information Officer (CIO) (Normenausschuss Maschinenbau im DIN 2007, S. 4), die Herausforderung von Methoden und Modellen darin, relevante Informationen in einem kognitiv verarbeitbaren Umfang zur Verfügung zu stellen. Die zuvor aufgeführten EAM-Frameworks integrieren Methoden und Modelle für EADesign und -Entwicklung und definieren entsprechende Metamodelle, respektive Vokabulare zur Sicherstellung einer intersubjektiv-nachvollziehbaren Beschreibung der Modelle (The Open Group 2009; Winter und Fischer 2007). Ein umfassendes Metamodell der EA-Entitäten aus Sicht eines Krankenhauses wird beispielsweise in (Mettler et al. 2008) beschrieben. Eine entsprechende Adaption auf die jeweiligen Concerns der Stakeholder einer Organisation wird jedoch meist nicht methodisch unterstützt. Im Anschluss an eine kurze Erläuterung der Eigenschaften von Krankenhäusern als komplexe Organisationen wird deshalb ein Metamodell auf Grundlage der Stakeholder Concerns in Krankenhäusern definiert, welches auf die fachlichen Fähigkeiten der Softwarearchitektur fokussiert, um eine Analyse des Beitrags zur Geschäftsarchitektur zu unterstützen.

\subsection{Krankenhäuser als komplexe Organisationen}

In Krankenhäusern werden Leistungen zur Heilung und Linderung von akuten Krankheiten, zur Gesundheitsprävention oder zur dauerhaften Versorgung chronisch kranker Personen von verschiedenen, hoch spezialisierten Berufsgruppen erbracht. Die Strukturen und Verfahren der Krankenhausorganisation kombinieren in einer, an den Berufsgruppen orientierten, funktional strukturierten Aufbauorganisation die ärztlichen Leistungen unterschiedlicher Disziplinen, bspw. Chirurgie oder Innere Medizin, mit denen der Pflege und den teilweise hoch spezialisierte Leistungen wie der Intensivpflege oder der Anästhesie sowie den medizinischen Querschnittsleistungen des Labors, der Pharmazie oder der Radiologie.

Im Vergleich zu anderen Industrien oder Organisationstypen integriert das System Krankenhaus in den Kern- und Unterstützungsprozessen ähnlich hoch 
spezialisierte Berufsgruppen, die jedoch stark unterschiedliche Ausbildungen, Anreize und Wertesysteme haben (Glouberman und Henry 2001, S. 67). Deshalb besteht eine große Herausforderung von Veränderungsprojekten in Krankenhäusern, die verschiedene Berufsgruppen betreffen, darin, die unterschiedlichen Perspektiven und Prioritäten der verschiedenen Stakeholder zu integrieren (Braun von Reinersdorff 2007, S. 41 ff.; Zelman et al. 2003, S. 1). Die Umsetzung von EAM in den Krankenhäusern und die entsprechende Reife von IS ist vergleichsweise gering (Hillestad et al. 2005, S. 1103 f.). Auf Grund diverser aktueller und zukünftiger Veränderungsanreize in den europäischen Gesundheitssystemen, wie beispielsweise der Orientierung weg von den Berufsgruppen hin zu einer patientenzentrierten Leistungserstellung und der Förderung grenzüberschreitender Kooperationen (Health and Consumers Directorate-General of the European Commission (Ed.) 2008), besteht insbesondere für Krankenhäuser die Herausforderung, sich strategisch zu positionieren und notwendige operative Änderungen innerhalb der Organisation entsprechend abzubilden.

Da nahezu jede Handlung im Gesundheitswesen mit Informationen verbunden ist (Porter und Olmsted Teisberg 2006, S. 208), leisten IS im Gesundheitswesen (HIS) als soziotechnische Systeme, d. h. als Komponenten und Prozeduren zur Generierung von Informationen, die fachliche Aktivitäten und Entscheidungen auf allen Ebenen des Gesundheitssystems verbessern (Lippeveld et al. 2000), einen wesentlichen Beitrag für die erfolgreiche Umsetzung notwendiger Veränderungen der Geschäftsarchitektur eines Krankenhauses. Die CIOs der Krankenhäuser sind deshalb besonders gefordert, die Veränderungen in der Geschäftsarchitektur entsprechend in der Softwarearchitektur abzubilden, um ein strategisches Alignment mit der Strategie der Organisation und den jeweiligen organisatorischen Ziele zu erreichen. Es bedarf also einer ganzheitlichen Bewertung von existierenden fachlichen Fähigkeiten der IT-Architektur, da einzelne Veränderungen innerhalb der ITArchitektur in Lösungssilos resultieren (Lorenzi et al. 2008, S. 292), die lediglich lokale Optimierungen ermöglichen und keinen organisationsweiten Beitrag leisten können.

\section{Metamodell-basierte Adaption von EAM Frameworks}

Um strategische und organisatorische Ziele sowie deren Auswirkungen auf die Geschäftsarchitektur adäquat in Integrationsarchitektur und Softwarearchitektur abzubilden, beschreibt Ross (2003) ein Vorgehensmodell, welches drei Schritte umfasst:

- Identifikation der strategischen Ziele der Organisation

- Definition der zentralen IT Fähigkeiten, um diese Ziele zu unterstützen

- Definition eines Vorgehens und entsprechende Auswahl technischer Komponenten, um die fachlichen Fähigkeiten der IT weiter zu entwickeln 
Dem Methodenengineering folgend (Bucher et al. 2007; Gutzwiller 1994) muss ein entsprechendes Methodenartefakt zur Modellierung der fachlichen Fähigkeiten der Softwarearchitektur im Rahmen eines Metamodells die relevanten Concerns der einzelnen fachlichen Stakeholder abbilden (Buckl et al. 2007; Kurpjuweit und Winter 2007; Lagerström et al. 2009). Somit kann sichergestellt werden, dass die einzelnen Aktivitäten der Methode entsprechende Ergebnisse produzieren, welche Aspekte von besonderem Interesse (,matter of interest") (Sutton und Rouvellou 2001, S. 1) berücksichtigen. Eine entsprechende Methode zum Viewpoint-based Meta Model Engineering (Kurpjuweit und Winter 2007) beschreibt fünf Schritte zur Identifikation von Metamodellen entsprechend der Concerns der Stakeholders der betrachteten Domäne, sogenannter Viewpoints. Die i) Identifikation relevanter Concerns bildet die Grundlage für eine ii) Anforderungsanalyse bezüglich des Informationsbedarfs, um die Concerns entsprechend zu adressieren. Auf dieser Basis wird iii) ein Überblick der Relationen der einzelnen Viewpoints realisiert, um iv) die einzelnen Metamodell-Entitäten zu modellieren und v) zu integrieren. Auf Basis bestehender generischer EAM-Frameworks können somit Metamodellerweiterungen oder -adaptionen entwickelt werden, welche die Anpassung der generischen Frameworks auf Basis der Stakeholder Concerns ermöglichen.

\section{Anforderungen an ein Metamodell zur Modellierung der IT- Architekturen in Krankenhäusern auf Grund von Stakeholder Concerns}

Als Folge der bisherigen Berufsgruppenorientierung und der mangelhaften Umsetzung einer Prozessarchitektur ist die Softwarearchitektur in Krankenhäusern durch eine starke Fragmentierung und Heterogenität (Khoumbati und Themistocleous 2006; Münz et al. 2007) charakterisiert. Aktuelle Trends des Gesundheitswesens, wie die Etablierung klinischer Behandlungspfade (De Bleser et al. 2006), die im Sinne von Referenzprozessen medizinische und pflegerische Abläufe definieren, um einen effizienten und koordinierten Ablauf sicherzustellen (Anderson et al. 1994), und das Konzept integrierter Behandlungseinheiten (Porter und Olmsted Teisberg 2006, S. 167 f.), fördern die organisationsinterne prozessuale, respektive organisatorische Koordination fachlicher Funktionen und resultieren in einer Prozessorientierung von Krankenhäusern.

Hieraus ergeben sich aus der Geschäfts- und Prozessarchitektur zwei grundsätzliche Stakeholder-Gruppen, die fachliche Führung und die operativen Akteure der einzelnen Berufsgruppen, und entsprechende Concerns. Einerseits streben fachliche Führungsebenen nach Integration und einer möglichst breiten funktionalen Abdeckung/Unterstützung der wertschöpfenden Aktivitäten der Organisation durch die Softwarearchitektur (Grimson et al. 2000, S.50; Raghupathi und Tan 2002, S. 57 ff.). Auf der operativen Ebene der Softwarenutzer werden die Concerns auf Grund des sozio-technischen Charakters von HIS determiniert. 
Hierbei hängt der Nutzen von Softwarearchitekturen stark von der Gesamtqualität der Interaktion zwischen Mensch, IT-System und Aufgabe ab (Ferstl und Sinz 2006, S. 67), da diese die Akzeptanz und Nutzung von Softwarekomponenten auf individueller Ebene beeinflussen (Anderson 1997; Ash et al. 2003). Entsprechend lassen sich für den Informationsbedarf der Modellierung der Softwarearchitektur in Krankenhäusern folgende Anforderungen definieren:

A1) Aus Sicht der fachlichen Führungsebene bedarf es einer Abbildung der fachlichen Funktionalitäten der Softwarearchitektur, die eine Auswertung des durch Software unterstützen Aktivitätsumfang der Prozessarchitektur ermöglichen (Brigl et al. 2005).

A2) Bezüglich der Breite der Verfügbarkeit der durch die Softwarearchitektur realisierten Funktionalitäten bedarf es einer Dokumentation der Nutzung innerhalb der Organisation (The Open Group 2009, S. 75).

A3) Bezüglich der effizienten und effektiven Umsetzung von Prozessen und Arbeitsabläufen bedarf es einer Dokumentation der Heterogenität der Softwarearchitektur bezüglich der fachlichen Funktionalitäten, da die redundante Ko-Existenz von Abläufen in verschiedenen Softwarekomponenten zu Inkonsistenzen in den Abläufen führt (West 2001).

A4) Bezüglich der individuellen Akzeptanz der Softwarenutzer bedarf es einer Analyse der Reife der durch die Softwarekomponenten realisierten fachlichen Funktionalitäten, da diese den Nutzen für die einzelnen Stakeholder beeinflussen (The Open Group 2009, S. 75).

\section{Metamodell zur Modellierung der fachlichen Funktionalitäten der Softwarearchitektur in Krankenhäusern}

Aus der in A1 definierten Anforderung ergeben sich typische Fragestellungen der Integrationsarchitektur. Für die Analyse respektive das Design der durch Software unterstützten Aktivitäten der Geschäftsprozesse bedarf es einer Modellierung der Geschäftsapplikationen, d. h. IS, die eine logische Gruppierung fachlicher Funktionalitäten von Softwarekomponenten darstellen. Die folgenden Ausführungen bilden die Grundlage für eine Erweiterung des zuvor genannten generischen Metamodells der EA-Entitäten aus Sicht eines Krankenhauses (Mettler et al. 2008), um entsprechend der Concerns und Anforderungen eine entsprechende Modellierung zu ermöglichen (vgl. Abbildung 1). Die Grundlage einer solchen Modellierung bildet eine funktionale Dekomposition der durch IT unterstützbaren Aktivitäten. Die funktionale Dekomposition wird im Krankenhaus ebenfalls im Rahmen der Organisationsgestaltung angewendet. Sie ermöglicht die Identifikation diskreter Funktionen einer Organisation im Rahmen derer die einzelnen Komponenten der komplexen Leistungserstellung in möglichst homogenen Gruppen zusammengefasst werden (The Open Group 2009, S. 426). Diese homogenen Gruppen können 
wiederum im Zuge der Prozess- oder Patientenorientierung für die Leistungserstellung entsprechend assembliert werden.

Um die fachlichen Funktionen der Softwarearchitektur zu identifizieren und entsprechend der Prozessarchitektur zuordnen zu können, beschreiben (Aier und Winter 2009) das Konzept einer Alignment-Architektur, welche mittels sogenannter fachlicher Services die einzelnen Komponenten der Softwarearchitektur (und gegebenenfalls der Technologiearchitektur) mit den Aktivitäten der Prozessarchitektur in Beziehung setzt. Fachliche Services repräsentieren hierbei logische Funktionsbündel von Softwarekomponenten (Schelp und Winter 2007, S. 5), die auf Grund der gemeinsamen Unterstützung von Geschäftsprozessen (fachliche Prozessservices), der gemeinsamen Bewirtschaftung von Informationsobjekten (fachliche Querschnittsservices) oder der gemeinsamen Wiederverwendung von Funktionalitäten (kanalorientierte fachliche Services) gruppiert werden (Schelp und Winter 2008, S. 13). In Anbetracht der zuvor beschriebenen hohen funktionalen Spezialisierung der Krankenhausmitarbeitenden und der Fokussierung auf die Wiederverwendbarkeit und Integration von IS-Funktionalitäten erscheinen kanalorientierte fachliche Services als besonders nützlich. Diese fachlichen Services gruppieren somit fachliche Funktionalitäten der Softwarekomponenten entsprechend der Aktivitäten und Funktionen der Berufsgruppen eines Krankenhauses. Entsprechende Referenzkataloge fachlicher Funktionalitäten der Softwarekomponenten eines Krankenhaus wurden bereits in Praxis und Wissenschaft identifiziert und bilden die Grundlage für eine Definition eines entsprechenden Vokabulars (Ammenwerth et al. 2002; Brigl et al. 2005; Department of Medical Informatics - University of Heidelberg 2001; Herbert 1993). Aus den Anforderungen A2 und A3 folgt einerseits die Notwendigkeit der Dokumentation, welche Softwarekomponenten die fachlichen Funktionalitäten realisieren, um Schlüsse auf die Heterogenität der Softwarearchitektur zuzulassen. Anderseits ermöglicht die Abbildung der Nutzung der fachlichen Funktionalitäten in den verschiedenen Geschäftsprozessen eine Analyse der Breite des Einsatzes der fachlichen Funktionalitäten. Wie in Abbildung 1 dargestellt, resultieren die in A2 und A3 definierten Anforderungen somit in Verfeinerungen des Metamodells durch die Darstellung der Relationen zwischen Geschäftsprozessen und fachlichen Funktionalitäten, sowie zwischen fachlichen Services und Softwarekomponenten. Die Anwendung der funktionalen Dekomposition im Rahmen der Organisationsgestaltung resultiert in homogenen Anforderungen in Bezug auf die Unterstützung einzelner Aufgaben der Prozesse und der entsprechenden ausführenden Rollen. Bezüglich A4 ermöglicht eine entsprechende Zuordnung fachlicher Funktionalitäten zu fachlichen Anforderungen somit eine Analyse des Nutzens der einzelnen Akteure des Krankenhauses. Zusammenfassend bildet das beschriebene Metamodell die Grundlage der Modellierung der fachlichen, funktionalen Fähigkeiten („Business Capabilities“) (The Open Group 2009, S. 488 f.) der Softwarearchitektur. Diese ermöglicht es, bestehende und anzustrebende Fähigkeiten eines Krankenhauses zu bewerten und bildet die Grundlage typischer IS-bezogener Analysen wie beispielsweise Abdeckungsanalysen auf 
Basis der identifizierten Stakeholder Concerns, um z. B. Lücken oder Redundanzen in der IT-Unterstützung von Geschäftsprozessen zu identifizieren (Aier et al. 2008b, S. 29).

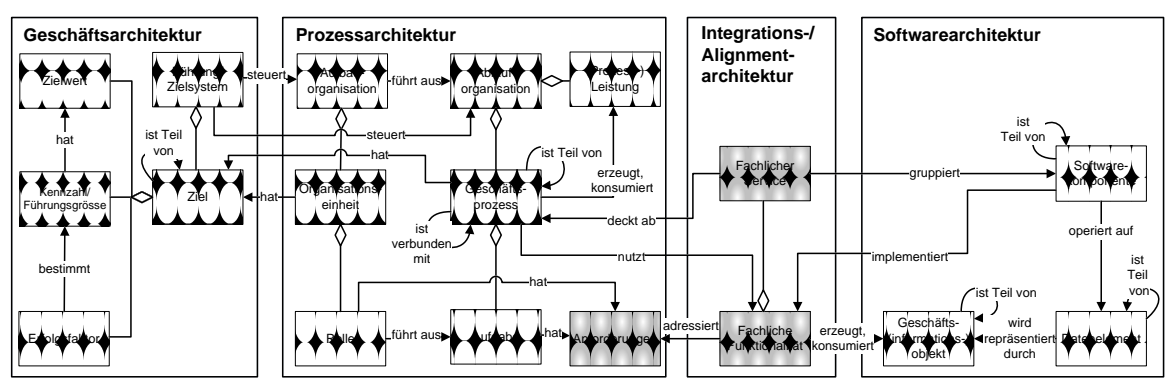

Abbildung 1: Metamodell zur Modellierung der fachlichen, funktionalen Fähigkeiten der Softwarearchitektur in Krankenhäusern, in Anlehnung an (Mettler et al. 2008)

\section{Konklusion}

Das in Abbildung 1 dargestellte Metamodell beschreibt die relevanten Entitäten der Modellierung einer EA in Krankenhäusern entsprechend der in Abschnitt 4 beschrieben Stakeholder Concerns. Es ermöglicht eine einheitliche Dokumentation der realisierten fachlichen Funktionalitäten auf Basis eines Referenzkatalogs von fachlichen Funktionalitäten (vgl. Abschnitt 5). Eine entsprechende Methode für eine vergleichende Abdeckungs- und Nutzenanalyse der Softwarearchitekturen wird momentan im Rahmen eines Benchmarks von 13 Schweizer Krankenhäusern entwickelt. Die fachlichen Fähigkeiten der Softwarearchitektur werden hierbei zur Krankenhaus-Wertkette (Porter und Olmsted Teisberg 2006, S. 200 ff.) in Bezug gesetzt und somit wird eine Bewertung des Beitrags zu den primären und unterstützenden Aktivitäten des Krankenhauses ermöglicht. Erste Ergebnisse der Studie zeigen eine umfangreiche Unterstützung der administrativen operativen Aufgaben von Krankenhäusern (Rechnungswesen, Personalwirtschaft, etc.) sowie eine grundsätzliche Unterstützung medizinisch operativer Aufgaben (Behandlungsprozesse, etc.) und administrativer Planungs- und Steuerungsprozesse (Controlling, etc.). Im Bereich der medizinischen Planung und Steuerung (Therapie- und Behandlungsplanung/-steuerung, Qualitätsmanagement etc.) besteht jedoch noch erhebliches Entwicklunspotentiale. Aufbauend auf dieser Arbeit wird im Rahmen einer detaillierten Analyse der 13 erhobenen Softwarearchitekturen eine Methode zur Auswertung der EA-Modelle bezüglich der krankenhausindividuellen strategischen Ziele entwickelt, um eine Bewertung und Entwicklung strategischer HIS zu realisieren. 


\section{Literatur}

Aier S und Gleichauf B (2009) Towards a Sophisticated Understanding of Service Design for Enterprise Architecture. In: Feuerlicht G, und Lamersdorf W (Hrsg) Service-Oriented Computing - ICSOC 2008 Workshops, Berlin.

Aier S, Riege C und Winter R (2008a) Classification of Enterprise Architecture Scenarios - An Exploratory Analysis. Enterprise Modelling and Information Systems Architectures 3(1): 14-23.

Aier S, Riege C und Winter R (2008b) Unternehmensarchitektur -

Literaturüberblick und Stand der Praxis. Wirtschaftsinformatik 50(4): 292-304.

Aier S und Winter R (2009) Virtuelle Entkopplung von fachlichen und ITStrukturen für das IT/Business Alignment - Grundlagen, Architekturgestaltung und Umsetzung am Beispiel der Domänenbildung. Wirtschaftsinformatik 51(2): 175-191.

Ammenwerth E, Buchauer A und Haus R (2002) A requirements index for information processing in hospitals. Methods of Information in Medicine 41(4): 282-288.

Anderson JG (1997) Clearing the Way for Physicians' Use of Clinical Information Systems. Communications of the ACM 40(8): 83-90.

Anderson KL, Anderson L und Glanze W (1994) Mosby's medical, nursing, and allied health dictionary. Mosby, St. Louis, MO.

Ash JS, Stavri PZ, Dykstra R und Fournier L (2003) Implementing Computerized Physician Order Entry: The Importance of Special People. International Journal of Medical Informatics 69(2-3): 235 - 250.

Braun von Reinersdorff A (2007) Strategische Krankenhausführung - Vom Lean Management zum Balanced Hospital Management. Verlag Hans Huber, Bern.

Brigl B, Ammenwerth E, Dujat C, Gräber S, Große A, Häber A, Jostes C und Winter A (2005) Preparing strategic information management plans for hospitals: a practical guideline: SIM plans for hospitals: a guideline. International Journal of Medical Informatics 74(1): 51-65.

Buchanan RD und Soley RM (2002) Aligning Enterprise Architecture and IT Investments with Corporate Goals, Object Management Group, Needham, MA.

Bucher T, Klesse M, Kurpjuweit S und Winter R (2007) Situational Method Engineering - On the Differentiation of "Context" and "Project Type". In: Ralyté J, Brinkkemper S, und Henderson-Sellers B (Hrsg) Situational Method Engineering - Fundamentals and Experiences, Boston. 
Buckl S, Ernst AM, Lankes J, Schneider K und Schweda CM (2007) A Pattern based Approach for Constructing Enterprise Architecture Management Information Models. In: Oberweis A, Weinhardt C, Gimpel H, Koschmider A, Pankratius V, und Schnizler B (Hrsg) eOrganisation: Service-, Prozess-, Market-Engineering, Karlsruhe.

CIO-Council (1999) Federal Enterprise Architecture Framework Version 1.1. http://www.cio.gov/Library/documents details.cfm?id=Federal\%20Enterpris e\%20Architecture $\% 20$ Framework, $\% 20$ Version $\% 201.1 \%$ 20September $\% 20199$ 9\&structure $=$ Enterprise $\% 20$ Architecture $\&$ category $=$ Enterprise $\% 20$ Architectu re. Abruf am 29.09.2009.

De Bleser L, Depreitere R, De Waele K, Vanhaecht K, Vlayen J und Sermeus W (2006) Defining pathways. Journal of Nursing Management 14(7): 553-563.

Department of Medical Informatics - University of Heidelberg (2001)

Requirements Index for Information Processing in Hospitals, University of Heidelberg, Heidelberg.

Ferstl OK und Sinz EJ (2006) Grundlagen der Wirtschaftsinformatik. Oldenbourg, München, Wien.

Frank U (2000) Evaluation von Artefakten in der Wirtschaftsinformatik. In: Hänschel I, und Heinrich LJ (Hrsg) Evaluation und Evaluationsforschung in der Wirtschaftsinformatik. Oldenbourg.

Glouberman S und Henry M (2001) Managing the Care of Health and the Cure of Disease-Part I: Differentiation. Health Care Management Review 26(1): 56-69.

Goethals F, Snoeck M, Lemahieu W und Vandenbulcke J (2006) Management and enterprise architecture click: The FADEE framework. Information Systems Frontiers 8(Enterprise Architecture): 67-79.

Grimson J, Grimson W und Hasselbring W (2000) The SI Challenge in Health Care. Communications of the ACM 43(6): 48-55.

Gutzwiller TA (1994) Das CC RIM-Referenzmodell für den Entwurf von betrieblichen, transaktionsorientierten Informationssystemen. Physica, Heidelberg.

Health and Consumers Directorate-General of the European Commission (Ed.) (2008) Europe for Patients. http://health.europa.eu/efp. Abruf am 29.09.2009.

Herbert I (1993) The Common Basic Specification, version4.4, Information Management Group (IMG), United Kingdom National Health Service (NHS).

Hevner AR, March ST, Park J und Ram S (2004) Design Science in Information Systems Research. MIS Quarterly 28(1): 75-105. 
Hillestad R, Bigelow J, Bower A, Girosi F, Meili R, Scoville R und Taylor R (2005) Can Electronic Medical Record Systems Transform Health Care? Potential Health Benefits, Savings, And Costs. Health Affairs 24(5): 1103-1117.

Ifip-Ifac (1998) GERAM: Generalised Enterprise Reference Architecture and Methodology, Version 1.6.2.

http://www.cit.gu.edu.au/ bernus/taskforce/geram/versions/geram1-62/v1.6.2.html\# Toc422226431. Abruf am 29.09.2009.

Khoumbati K und Themistocleous M (2006) Integrating the IT Infrastructures in Healthcare Organisations: a Proposition of Influential Factors. Electronic Journal of e-Government 4(1): 27-36.

Kurpjuweit S und Winter R (2007) Viewpoint-based Meta Model Engineering. In: Reichert M, Strecker S, und Turowski K (Hrsg) Enterprise Modelling and Information Systems Architectures - Concepts and Applications, Proceedings of the 2nd Int'l Workshop EMISA 2007, Bonn.

Lagerström R, Saat J, Franke U, Aier S und Ekstedt M (2009) Enterprise Meta Modeling Methods - Combining a Stakeholder-Oriented and a Causality-Based Approach. In: Halpin T, Krogstie J, Nurcan S, Proper E, Schmidt R, Soffer P, und Ukor R (Hrsg) Enterprise, Business-Process and Information Systems Modeling, Proceedings of the 10th International Workshop BPMDS and 14th International Conference EMMSAD at CAiSE 2009, Amsterdam.

Lankhorst M (2005) Enterprise Architecture at Work: Modelling, Communication and Analysis. Springer, Berlin et al.

Lippeveld T, Sauerborn R und Bodart C (Hrsg) Design and Implementation of Health Information Systems (2000). World Health Organization, Geneva.

Lorenzi NM, Novak LL, Weiss JB, Gadd CS und Unertl KM (2008) Crossing the Implementation Chasm: A Proposal for Bold Action. Journal of the American Medical Informatics Association 15(3): 290-296.

March ST und Smith GF (1995) Design and Natural Science Research on Information Technology. Decision Support Systems 15(4): 251-266.

Mettler T, Rohner P und Baacke L (2008) Improving Data Quality in Health Information Systems - A Holistic Design-oriented Approach. In: Golden W, Acton T, Conboy K, van der Heijden H, und Tuuainen V (Hrsg) Proceedings of the 16th European Conference on Information Systems, Galway.

Münz JO, Müller L und Behavka P (2007) Integration and Management of Large Heterogeneous Healthcare Information Systems. In: Blobel B, Pharow P, Zvarova J, und Lopezs D (Hrsg) eHealth: Combining Health Telematics, Telemedicine, Biomedical Engineering and Bioinformatics to the Edge. IOS Press, Regensburg. 
Niemann KD (2005) Von der Unternehmensarchitektur zur IT-Governance: Leitfaden für effizientes und effektives IT-Management. Vieweg.

Normenausschuss Maschinenbau im DIN (Hrsg) DIN EN ISO 19439:2006 Unternehmensintegration - Rahmenwerk für Unternehmensmodellierung (2007). Deutsches Institut für Normung e.V., Berlin.

Porter ME und Olmsted Teisberg E (2006) Redefining Health Care: Creating Value-Based Competition on Results. Harvard Business School Press, Boston, MA.

Raghupathi W und Tan J (2002) Strategic IT applications in health care. Communications of the ACM 45(12): 56-61.

Ross JW (2003) Creating a strategic IT architecture competency: Learning in stages. Mis Quarterly Executive 2(1): 31-43.

Schelp J und Winter R (2007) Towards a Methodology for Service Construction. In: Sprague RH (Hrsg) Proceedings of the 40th Hawaii International Conference on Systems Sciences (HICSS-40), Waikoloa, Big Island, HI

Schelp J und Winter R (2008) Entwurf von Anwendungssystemen und Entwurf von Enterprise Services - Ähnlichkeiten und Unterschiede. Wirtschaftsinformatik 50(1): 6-15.

Sutton SM und Rouvellou I (Hrsg) Issues in the Design and Implementation of a Concern-Space Modeling Schema (2001). Toronto, Canada.

The Open Group (Hrsg) TOGAF Version 9 - The Open Group Architecture Framework (2009). The Open Group, San Francisco, CA.

Veasey PW (2001) Use of enterprise architectures in managing strategic change. Business Process Management Journal 7(5): 420-436.

West E (2001) Management matters: the link between hospital organisation and quality of patient care. Quality in Health Care 10(1): 40-48.

Winter R und Fischer R (2007) Essential Layers, Artifacts, and Dependencies of Enterprise Architecture. Journal of Enterprise Architecture 3(2): 7-18.

Zachman JA (1987) A Framework for Information Systems Architecture. IBM Systems Journal 26(3): 276-292.

Zelman WN, Pink GH und Matthias CB (2003) Use of the Balanced Scorecard in Health Care. Journal of Health Care Finance 29(4): 1-16. 